experimented in ultrasound. Five enthesis locations bilaterally (distal Achilles tendon, distal andproximal patellar ligaments, distal quadriceps, and brachial triceps tendons) in each person were explored.The following elemental lesions of enthesis were evaluated: thickness, presence of calcifications, erosions, enthesophyte, loss of fibrillar patternand power Doppler signal.

The calculated index was compared by Mann-Whitney $U$ test between cases and controls. The significance level was set at $5 \%$.

Results: In our study population, the median age was $51.8 \pm 2.3$ years and the median body mass index was $30 \pm 1 \mathrm{~kg} / \mathrm{m}^{2}$. This last was similar between the two groups. All included subjects were female.

The total enthesitis index was higher in G1 $(6.67 \pm 0.91)$ than G2 $(3.50 \pm 0.73)$ with a statistically significant difference $(p=0.01)$.

Considering each evaluated enthesis, the distal patellar ligament score was significantly higher in the $\mathrm{G} 1(1.67 \pm 0.55$ vs $0.25 \pm 0.16$ with $p=0.03)$. For the other enthesis, there was no significantly difference between the 2 groups.

Conclusions: The distal patellar ligament enthesis changes shown in older persons may be the traduction of a silent-stage of knee osteoarthritis.

Disclosure of Interest: None declared

DOI: 10.1136/annrheumdis-2017-eular.6630

\section{AB1039 RELATIONSHIPS BETWEEN SONOGRAPHIC AND ELECTROPHYSIOLOGICAL MEASURES IN PATIENTS WITH IDIOPATHIC CARPAL TUNNEL SYNDROME WAITING FOR SURGERY}

M. Valls Roc ${ }^{1}$, E. Junyent Vilanova ${ }^{2}$, A. Grau Martin ${ }^{3}$, O. Codina Guinó ${ }^{1}$, M. Sala Gomez ${ }^{1} .{ }^{1}$ Rheumatology; ${ }^{2}$ Traumatology; ${ }^{3}$ Internal Medicine, HOSPITAL of FIGUERES, Girona, Spain

Background: Sonography is a diagnostic tool with great development in diagnosing entrapment neuropathy. It's an easy, painless, fast, non-invasive technique and can explore how the nerve's morphology and pathologies are associated. An electroneurogram is used to assess the intensity of nerve involvement.

Objectives: To determine the relationship between the intensity of nerve involvement by electroneurogram and the measurement of the cross-sectional area (CSA) of the median nerve by sonography in patients with idiopathic carpal tunnel syndrome (CTS) waiting for surgery.

Methods: 56 wrists of 39 consecutive patients waiting for surgery were tested, however 5 were excluded because were found to have anatomic variants ( 4 bifid nerves, 2 median arteries) and 1 fybrolipoma. Therefore, the final sample was 51 wrists of 37 consecutive patients (11 male and 26 females), with a mean age of 59.2 years (26-85), all with electrophysiologically confirmed idiopathic CTS. Patients were classified by their electrophysiologic grade. The median nerve cross-sectional area at proximal and distal carpal tunnel was measured using high frequency ultrasound.

Relationships between CSA, the severity of the electrophysiologic grade and the duration of symptoms were analysed. Also, a median nerve morphological characteristics examination (hipoecogenicity, loss of fascicular structure, Power Doppler signal and anatomical variants) was undertaken.

A comparison between CSA and the severity of the electrophysiologic grade was made using an independent $T$ test and the connection between CSA and the duration of symptoms was calculated using ANOVA test.

Results: Patients were classified by their electrophysiologic severity grade (8 mild, 13 moderate, 29 severe and 1 very severe). The mean ultrasound area of distal medial nerve was $8.7 \mathrm{~mm}^{2}$ in mild-moderate and $9.2 \mathrm{~mm}^{2}$ in severe-very severe cases $(p=0.52)$. The average of proximal CSA was $11.6 \mathrm{~mm}^{2}$ in mild-moderate and $14.1 \mathrm{~mm}^{2}$ in severe-very severe cases with statistical signification differences $(p=0.026)$. Relationship between CSA and symptom's duration wasn't identified. In $89.2 \%$ of the cases, hipoecogenicity and the loss of fascicular structure were observed but no cases were found to show positive Power Doppler signal.

Conclusions: The most valid and relevant parameter regarding the electroneurogram in the diagnosis of CTS is CSA at proximal carpal tunnel by sonography. A cross-sectional area measuring more than $9-10 \mathrm{~mm}^{2}$ has been suggested to be pathologic and our study confirms these results. While the electroneurogram is the gold-standard method in the diagnosis of nerve involvement severity, a sonography could improve the diagnostic sensibility and give information about nerve's morphology and associated pathologies.

Disclosure of Interest: None declared

DOI: 10.1136/annrheumdis-2017-eular.4214

\section{AB1040 CLINICAL UTILITY OF BONE SCINTIGRAPHY FOR INFLAMMATORY ARTHRITIS}

M. Jiang, K. Le Marshall, K. Lim. Department of Rheumatology, Western Health, Footscray, Australia

Background: Bone scintigraphy is often used in the workup of patients with rheumatological disease, in particular for the investigation of inflammatory arthritis. It also has a role in the investigation of malignancy and fractures. As an imaging technique, it is very sensitive but not specific for inflammation. The most common technique used is triple phase scintigraphy, with the 2 nd phase (blood pool phase) being the most useful for identifying inflammation.
Objectives: To evaluate the clinical utility of bone scintigraphy in the workup of patients with rheumatological disease, in particular for inflammatory arthritis.

Methods: This was a retrospective study of patients seen in the rheumatology outpatients between January 2011 and July 2014, who had bone scintigraphy as part of their workup. Their clinical record was reviewed to obtain pre- and posttest clinical diagnoses, bone scintigraphy reports and investigations (ESR/CRP, rheumatoid factor/CCP antibodies). For patients who had followup at one year we recorded their clinical diagnosis at this time.

Results: A total of 226 patients had bone scintigraphy, with a median age of 54 years. $63 \%$ were female.

The main indication for bone scintigraphy was to assess for inflammation in 194 patients. For this group, the most common pre-test diagnosis of inflammatory arthritis $(41 \%)$, followed by degenerative arthritis $(36 \%)$, unclear diagnosis $(20 \%)$ and mixed inflammatory and degenerative arthritis (3\%).

Overall, $49 \%(n=95)$ of patients had their diagnosis changed after bone scintigraphy.

The pre-test diagnosis was compared to bone scintigraphy findings with the highest confirmatory rate for degenerative arthritis $(67 \%)$, followed by inflammatory arthritis (49\%) and mixed arthritis (40\%).

Bone scintigraphy findings were also compared to post test diagnosis with the highest confirmatory rate for degenerative arthritis $(91 \%)$, followed by inflammatory arthritis (70\%) and mixed arthritis (14\%).

There was no significant association between patient factors (age, gender, ESR/CRP, RF/CCP) and having confirmatory or conflicting bone scintigraphy findings.

The post test diagnosis was compared to the diagnosis at one year, with the diagnosis being unchanged in $84 \%$ for inflammatory arthritis and $45 \%$ for degenerative arthritis.

Conclusions: This study showed that bone scintigraphy lead to a change in diagnosis in a large proportion of patients and was better at confirming degenerative arthritis or ruling out inflammatory arthritis.

References:

[1] De Leonardis F, Orzincolo C, Prandini N, Trotta F. The role of conventional radiography and scintigraphy in the third millennium. Best practice \& research Clinical rheumatology. 2008;22(6):961-79.

[2] Kim JY, Cho SK, Han M, Choi YY, Bae SC, Sung YK. The Role of Bone Scintigraphy in the Diagnosis of Rheumatoid Arthritis According to the 2010 ACR/EULAR Classification Criteria. Journal of Korean Medical Science. 2014;29(2):204-9.

[3] Sudoł-Szopińska I, Ćwikła JB. Current imaging techniques in rheumatology: MRI, scintigraphy and PET. Polish Journal of Radiology. 2013;78(3):48-56.

[4] Al-Janabi M. Imaging modalities of the painful wrist: the role of bone scintigraphy. Rheumatology (Oxford, England). 2002;41(10):1085-7.

Acknowledgements: I would like to acknowledge Sara Vogrin (University of Melbourne) for her assistance with statistical analysis.

Disclosure of Interest: None declared

DOI: 10.1136/annrheumdis-2017-eular.4591

\section{AB1041 ULTRASONOGRAPHY OF NORMAL MUSCULOSKELETAL STRUCTURES IN 100 SECTIONS: A BOOKLET AND A CD-ROM}

M.A. Mahdi, H. Rkain, M. Erraoui, S. Aktaou, L. Tahiri, R. Bahiri, F. Allali, N. Hajjaj-Hassouni. Rheumatology, Faculty of Medicine and Pharmacy, University Mohamed V, Rabat, Morocco

Objectives: To present a booklet and a CD-ROM with a mini-atlas including 100 sections illustrating the normal ultrasound musculoskeletal anatomy.

Methods: We performed an ultrasound examination of large and small joints of the medical staff not suffering from any musculoskeletal disorder. Ultrasound examination was performed using a high-frequency linear probe (Toshiba Xario ${ }^{\circledR}$, frequency ( $8-14 \mathrm{MHz})$ ) in $\mathrm{B}$ mode. Finally, for the sake of clarity of the presentation of this library, we presented each image accompanied with another showing the valid positioning of the probe and an annotated schema for each section made. Results: We present in the form of CD-ROM and booklet a photo library of a mini-atlas.

\section{Section 27 ; Cross-section of the flexor digitorum superficialis and} profondus tendons
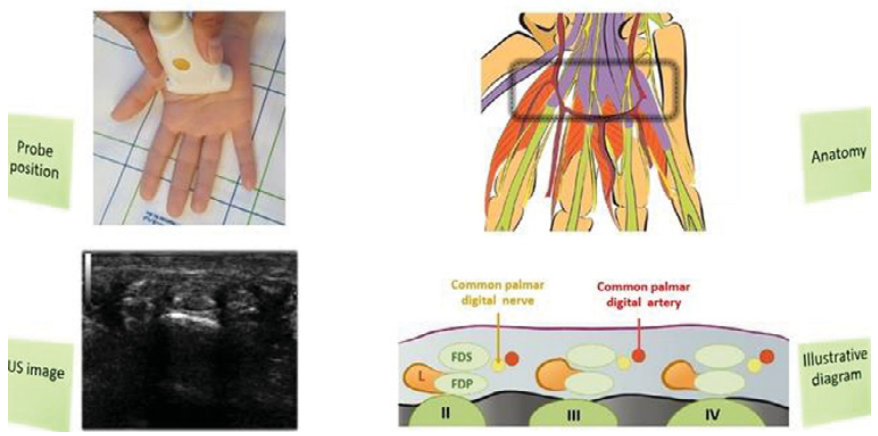